\title{
Numerical optimisation of operating conditions of waste to energy
}

\author{
M. Kapitler, F. Kokalj \& N. Samec \\ Faculty of Mechanical Engineering, University of Maribor, Slovenia
}

\begin{abstract}
The combustion process, using municipal solid waste (MSW) as a fuel in waste to energy plant, calls for a detailed understanding of these phenomena. On the one hand, this process depends on many input parameters, like MSW proximate and ultimate analysis, the season of the year, primary and secondary inlet air velocity, and on the other hand on the output parameters such as temperature or mass flow rate (MFR) of combustion products on the exhaust.

The variability and mutual dependence of these parameters can be difficult to manage in practice. Moreover, another problem is how these parameters can be tuned to achieve the optimal combustion with minimal pollutants emission during the plant design phase already. To meet these goals, waste to energy plant with bed combustion is investigated by using computational fluid dynamics (CFD) approach with ANSYS CFX 12.0 code in the WORKBENCH 2 environment. In this paper, the adequate variable input boundary conditions which are based on the real measurement and practical calculations of known MSW composition from other authors are used and the whole computational work is updated with real plant geometry and the appropriate turbulence, combustion and heat transfer models. Furthermore, the operating parameters were optimized on output parameters through trade-off study. Different operating conditions were varied and the fluid flow direction, residence time, temperature field, velocity field, nitric oxide formation and combustion products through plant combustion chamber and preheat intersection in $3 \mathrm{D}$ were predicted and visualized. The optimization in real time has shown the amount of each input parameters to meet the optimal operating conditions. Finally, the response charts between the input and output parameters are presented to monitor the dependence among these parameters. Further simulations have to be done to include the geometry dimensions as input parameters.
\end{abstract}

Keywords: municipal solid waste (MSW) modelling, bed combustion, 
computational fluid dynamics (CFD), operating conditions, numerical optimisation, trade-off study, parameter sensitivity, response charts.

\section{Introduction}

Nowadays, bed combustion on grate is the most common way to burn municipal solid waste (MSW) in waste to energy plants (WTEP). The combustion in these plants are very specific due to the characteristics of fuel (MSW) which depend on proximate and ultimate analyze, season of the year, primary and secondary inlet air velocity and many other parameters which changes all the time in some frames. The goals are how we can tune the optimal combustion with minimal emission of the pollutants in the environment already in the plant project phase. To meet these goals, WTEP with bed combustion is investigated by using computational fluid dynamics (CFD) approach with ANSYS CFX 12.0 code in the WORKBENCH 2 environment. By using this tool the input and output parameters can be followed and visualized the mutual interaction. The mathematical models were developed like FLIC [4, 7] and test project TAMARA [11] to find out combustion products distribution in the gaseous phase along the travel bed. The combustion of MSW in the gaseous phase combustion has many phases like moisture evaporation, waste devolatilisation, combustion of volatiles, mixing and fixed carbon combustion during heterogeneous chemical reactions [1].

\section{Numerical optimization of operating conditions}

\subsection{Models description}

The combustion in a WTEP is described quite accurately by a system of differential equations of the reacting flow so called Reynolds Averaged NavierStokes equations (RANS), presented in the following form:

$$
\begin{gathered}
\frac{\partial \bar{\rho}}{\partial t}+\frac{\partial}{\partial x_{j}}\left(\bar{\rho} \bar{v}_{j}\right)=0 \\
\frac{\partial}{\partial t}\left(\bar{\rho} \bar{v}_{j}\right)+\frac{\partial}{\partial x_{j}}\left(\bar{\rho} \bar{v}_{j} \bar{v}_{i}\right)=-\frac{\partial p}{\partial x_{i}}+\overline{f_{v i}}-\frac{\partial}{\partial x_{j}}\left(\bar{\tau}_{i j}+\overline{\rho v_{j}^{\prime} v_{i}^{\prime}}\right) \\
\frac{\partial}{\partial \mathrm{t}}(\bar{\rho} \overline{\mathrm{h}})+\frac{\partial}{\partial \mathrm{x}_{\mathrm{j}}}\left(\bar{\rho} \bar{v}_{\mathrm{j}} \overline{\mathrm{h}}\right)-\frac{\partial \mathrm{p}}{\partial \mathrm{t}}+\frac{\partial}{\partial \mathrm{x}_{\mathrm{j}}}\left(\overline{\mathrm{q}}_{\mathrm{j}}+\overline{\rho v_{\mathrm{j}}^{\prime} \mathrm{h}^{\prime}}\right)=\overline{\mathrm{I}_{\mathrm{T}}} .
\end{gathered}
$$

Reynolds' stresses $\left(\overline{\rho v_{j}^{\prime} v_{i}^{\prime}}\right)$ are modeled by the introduction of turbulent viscosity $\eta_{t}$ :

$$
\overline{\rho v_{j}^{\prime} v_{i}^{\prime}}=\frac{2}{3} \delta_{i j}\left(\rho k+\eta_{t} \frac{\partial v_{k}}{\partial x_{k}}\right)-\eta_{t}\left(\frac{\partial v_{i}}{\partial x_{j}}+\frac{\partial v_{j}}{\partial x_{i}}\right) .
$$


Turbulent viscosity can be determined using various turbulent models to close-down the system of Reynolds' equations. The two-equation $k-\varepsilon$ turbulent model is used for the purpose of the presented reacting flow modelling. Application of $k-\varepsilon$ turbulent model in the modelling of reacting flows has already been proven by many authors as a very successful one. Turbulent viscosity is computed using:

$$
\eta_{t}=\rho C_{\eta} \frac{k^{2}}{\varepsilon}
$$

where $k$ is turbulent kinetic energy $-k=0.5\left(\overline{v_{i}^{\prime} v_{i}^{\prime}}\right)$ and $\varepsilon$ its dissipation (irreversible transformation of kinetic energy into internal energy).

Local values of $k$ and $\varepsilon$ are computed using the following transport equations:

$$
\begin{aligned}
& \frac{\partial}{\partial t}(\rho k)+\frac{\partial}{\partial x_{j}}\left(\bar{v}_{j} k\right)-\frac{\partial}{\partial x_{j}}\left[\left(\eta+\frac{\eta_{t}}{\sigma_{k}}\right) \frac{\partial k}{\partial x_{j}}\right]=I_{k}, \\
& \frac{\partial}{\partial t}(\rho \varepsilon)+\frac{\partial}{\partial x_{j}}\left(\bar{v}_{j} \varepsilon\right)-\frac{\partial}{\partial x_{j}}\left[\left(\eta+\frac{\eta_{t}}{\sigma_{\varepsilon}}\right) \frac{\partial \varepsilon}{\partial x_{j}}\right]=I_{\varepsilon},
\end{aligned}
$$

the source terms are modelled as:

$$
\begin{gathered}
I_{k}=\eta_{t}\left(\frac{\partial \bar{v}_{i}}{\partial x_{j}}+\frac{\partial \bar{v}_{j}}{\partial x_{i}}\right) \frac{\partial \bar{v}_{i}}{\partial x_{j}}-\rho \varepsilon \\
I_{\varepsilon}=C_{1} \frac{\varepsilon}{k}\left[\eta_{t}\left(\frac{\partial \bar{v}_{i}}{\partial x_{j}}+\frac{\partial \bar{v}_{j}}{\partial x_{i}}\right) \frac{\partial \bar{v}_{i}}{\partial x_{j}}\right]-C_{2} \rho \frac{\varepsilon^{2}}{k} .
\end{gathered}
$$

Reynolds' enthalpy flux $\overline{\rho v_{i}^{\prime} h^{\prime}}$ in equation (3) is also defined with turbulent viscosity:

$$
\overline{\rho v_{j}^{\prime} h^{\prime}}=-\frac{\eta_{t}}{\operatorname{Pr}_{t}} c_{p} \frac{\partial T}{\partial x_{j}},
$$

where $\operatorname{Pr}_{t}$ is the turbulent Prandtl number. $C_{\eta}, C_{l}, C_{2}, \sigma_{k}$ and $\sigma_{\varepsilon}$ are constants, and their values used in the presented work are: $C_{\eta}=0,09 ; C_{1}=1,44 ; C_{2}=1,92$; $\sigma_{k}=1$ and $\sigma_{\varepsilon}=1,3$.

Advection - diffusive equation of mass species $\left(\xi_{k}\right)$ of the component $k$ has due to Reynolds' averaging, an additional term called turbulent mass species flux:

$$
\overline{\rho \xi_{k}^{\prime} v_{j}^{\prime}}=\frac{\eta_{t}}{\mathrm{Sc}_{t}} \frac{\partial \xi_{k}}{\partial x_{j}},
$$


and can be modelled with turbulent viscosity using the $k-\varepsilon$ model. The complete advection - diffusive mass species equation is:

$$
\frac{\partial}{\partial t}\left(\overline{\rho \xi_{k}}\right)+\frac{\partial}{\partial x_{j}}\left(\overline{\rho v_{j} \xi_{k}}\right)-\frac{\partial}{\partial x_{j}}\left[\left(\rho D_{k}+\frac{\eta_{t}}{\mathrm{Sc}_{t}}\right) \frac{\partial \xi_{k}}{\partial x_{j}}\right]=\overline{I_{\xi_{k}}},
$$

where $\mathrm{Sc}_{t}$ is the turbulent Schmidt number and $D_{k}$ molecular diffusion coefficient of component $k$. With the new term:

$$
\Gamma_{k, e f f}=\rho D_{k}+\frac{\eta_{t}}{\mathrm{Sc}_{t}}=\Gamma_{k}+\frac{\eta_{t}}{\mathrm{Sc}_{t}}
$$

the equation (12) can be rewritten as:

$$
\frac{\partial}{\partial t}\left(\overline{\rho \xi_{k}}\right)+\frac{\partial}{\partial x_{j}}\left(\overline{\rho v_{j} \xi_{k}}\right)-\frac{\partial}{\partial x_{j}}\left(\Gamma_{k, e f f} \frac{\partial \xi_{k}}{\partial x_{j}}\right)=\overline{I_{\xi_{k}}}
$$

Source terms of energy and mass species transport equations are computed by the following two equations where $\omega_{k}$ is computed by the turbulent combustion model:

$$
\begin{gathered}
\overline{I_{T}}=-\sum_{k=1}^{N} \Delta H_{f, k}^{o} \bar{\omega}_{k}, \\
\overline{I_{\xi_{k}}}=M_{k} \bar{\omega}_{k},
\end{gathered}
$$

where $\Delta H_{f, k}^{\circ}$ is the standard heat formation and $M_{k}$ the molecular mass of the component $k$. In equations (15) and (16) the $\omega_{k}$ stands for the formation/consumption rate of component $k$ and is defined by the following expression:

$$
\bar{\omega}_{k}=\frac{d\left[X_{k}\right]}{d t}=\left(v_{k}^{\prime \prime}-v_{k}^{\prime}\right) \bar{R}_{k}
$$

which is written for the general form of the chemical reaction:

$$
\sum_{k=1}^{N} v_{k}^{\prime} X_{k} \underset{k_{b}}{\stackrel{k_{f}}{\rightleftarrows}} \sum_{k=1}^{N} v_{k}^{\prime \prime} X_{k}
$$

where $v_{k}$ and $v^{\prime \prime}{ }_{k}$ designate the stoichiometric coefficients of component $k$ for reactants and products, respectively. Chemical reaction rate $R_{k}$ in equation (17) is calculated by appropriate combustion models. It has to be pointed out that nowadays many turbulent combustion models are in practical use. Their application depends on the type of combustion (diffusion, kinetic, mixed), fuel type (solid, liquid, gaseous) and combustion device (furnace, boiler, engine). Most of models include various empirical constants which need to be individually determined case by case. In this case the Eddy Dissipation Combustion Model has been applied. 


\subsection{Geometry, meshing and boundary conditions}

With Computational Fluid Dynamics (CFD) approach we can predict the combustion processes and to optimize the operating condition and combustion chamber in existing waste to energy power plant (WTEP) or in the project phase. Figure 1 shows the 2D engineering plan view of WTEP.

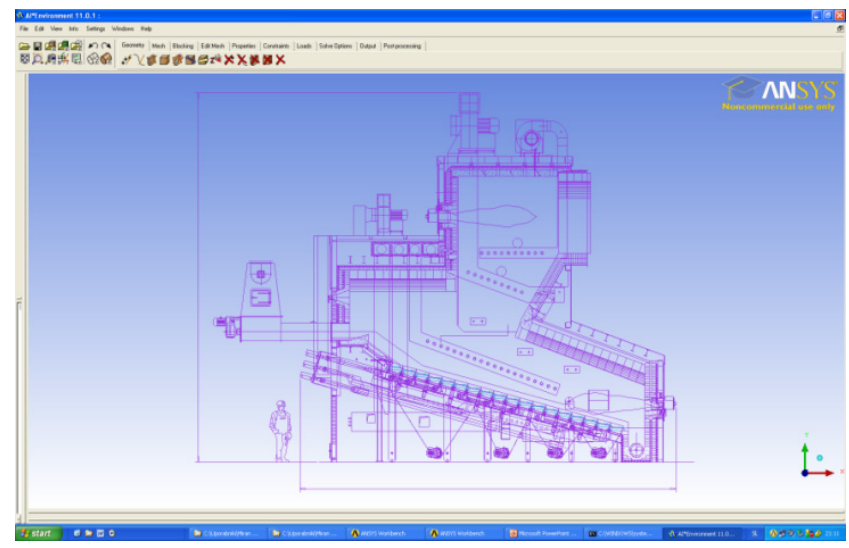

Figure 1: The 2D engineering plan view of WTEP.

On this base the WTEP was built and operates with RDF as a fuel produced with MBT from MSW. Figure 2 shows grate, details in the primary combustion chamber with waste input (left) and the secondary combustion chamber with secondary and tertiary air inlet (right).

The 3D geometry plan on the base of engineering plans in real measure was drown (Figure 3 - left). Each dimension was marked on the plan with corresponding input dimensions. In this way each dimension is easy and quickly modified and the entire construction can be quickly changed and redrawn.
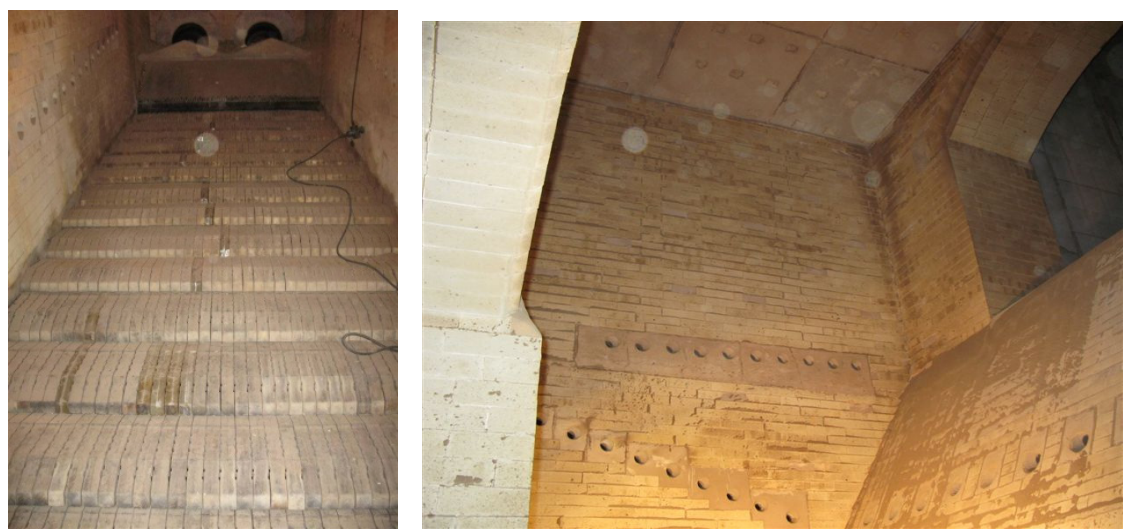

Figure 2: Grate in primary combustion chamber and secondary and tertiary air inlets. 
Furthermore, the meshing of ca. 1,4 million cells Figure 3 - right) is created and the boundary conditions with entire combustion, radiation, particle tracking and other models, input and output parameters are set up. These input parameters are operating conditions like input velocities, temperatures, reactants mass flow rates (MFR), dimension value and the output parameters like temperatures, combustion products MFR and other exhaust parameters. It is known that the concentration of gaseous components which are created from MSW combustion like carbon monoxide, methane, hydrogen, nitrogen monoxide or oxygen above the bed are different distributed along the grate set. These facts are taken into consideration when the boundary conditions are defined. In this study, the measured operating parameters form existing WTEP is used as the input parameters (IP). In addition, the reactions for gaseous combustion are also defined and the entire model is solved. Many other authors have used the FLUENT solver code but in this study it is interesting that the CFX V12.0 solver code in the Workbench 2 environment which was developed by Ansys, Inc. has been used and the results are quite similar.
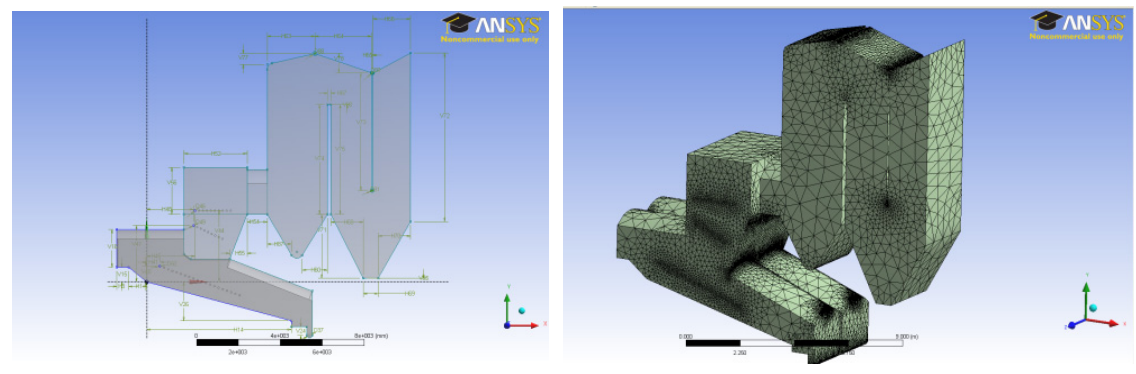

Figure 3: 3D geometry plan of WTEP with dimensions (left) and meshing (right).

The optimisation process was made by using design exploration which is a powerful tool for designing and understanding the analysis response of parts and assemblies. It is a deterministic method based on Design of Experiments (DOE) and various optimisation methods with parameters as its fundamental components. DOE techniques has one common characteristic: it try to locate the sampling points such that the space of random input parameters is explored in the most efficient way, or obtain the required information with a minimum of sampling points. Sample points in efficient locations will not only reduce the required number of sampling points, but also increase the accuracy of the response surface that is derived from the results of the sampling points. By default the deterministic method uses a central composite design, which combines one centre point, points along the axis of the input parameters, and the points determined by a fractional factorial design. GDO is a constrained, multiobjective optimisation technique in which the best possible designs are obtained from sample set given the objectives you set form parameters. GDO can be used for design optimisation in three ways: the screening, the Multi-Objective Generic Algorithm (MOGA) approach and the Non-Linear Programming by Quadratic 
Lagrangian approach (NLPQL). The screening approach is a non-iterative direct sampling method by a quasi-random number generator based on Hammersley algorithm. MOGA approach is an iterative Multi-Objective Genetic Algorithm, which can optimize problems with continuous input parameters. NLPQL is a gradient based single objective optimizer which is based on the mathematical optimisation algorithm developed by Klaus Schittkowski. GDO has more possible objectives and they are: no-objective, minimize, maximize, target value which is less than or equal to or greater than or equal to the input target value. The importance of the parameter is default, lower and higher. By setting these targets the three best candidates (A, B or C) are chosen which best met all the requests.

Probabilistic sensitivities are based on a statistical correlation analysis between the individual probabilistic design variables. You can review the parameters correlation data that has been used to derive sensitivities and decide if individual sensitivity values are significant or not. This information is collected in the correlation matrix of the random output parameters versus the random input variables. It can be also reviewed the correlations that have been sampled between random input variables. The correlations between random output parameters are important if you want to use the probabilistic results of your probabilistic analysis as input for another probabilistic analysis.

When the optimisation process is finished all results can be studied and the response charts can be visualized.

\section{Results and discussion}

In the study three input parameters (IP) are used: secondary air inlet velocity (InSecAirVelocity-m/s), oxygen MFR (MassInVelocity in $\mathrm{m} / \mathrm{s}$ ) and MWS velocity (MSWVelocity in $\mathrm{m} / \mathrm{s}$ ) and many other output parameters (OP): average value of following parameters at the exit of the WTEP - CO (COOut dimensionless), temperature (TempOut in Kelvin (K), $\mathrm{CH}_{4} \quad$ (CH4Out dimensionless), oxygen (O2Out dimensionless); average value ash travelling time along the grate (AshTime in s), average ash temperature (AshTemp in K), maximal ash temperature through the WTEP (AshMaxTemp in K), etc.

On the other hand, we have to be shore that the maximum ash temperature does not exceed the ash melting point and we have to avoid flying ash deposit on heat exchangers walls which can cause a great damage. The optimisation response chart (Figure 1:) which was created as results of DOE helps us to determinate the interaction among maximal ash temperature versus secondary air velocity and oxygen MFR in secondary air inlet. The maximal ash temperature from secondary air velocity of $27 \mathrm{~m} / \mathrm{s}$ to $29 \mathrm{~m} / \mathrm{s}$ increases rapidly and picked the maximum ash temperature at $1850 \mathrm{~K}$. On the other hand, there is no significant dependence of oxygen MFR in region from 0.255 to 0.21 . In this way we can predict and avoid the damage it can cause this phenomenon.

Table 1 shows setting and the results of trade-off optimisation with IP and OP. As we can see three candidates which best match the requirements appear with belonging values. In this way the optimisation is easy and effective used. 
38 Waste Management and the Environment V

Table 1: $\quad$ Trade-off study parameters objective settings and results.

\begin{tabular}{|c|c|c|c|c|c|c|c|c|c|c|c|c|}
\hline \multicolumn{13}{|c|}{ Table of Schematic B4: Optimization } \\
\hline$\checkmark$ & \multicolumn{2}{|c|}{ A } & \multicolumn{2}{|c|}{ B } & \multicolumn{2}{|r|}{$\mathrm{c}$} & D & \multicolumn{2}{|r|}{ E } & \multicolumn{2}{|r|}{$\mathrm{F}$} & G \\
\hline 1 & & & \multicolumn{2}{|c|}{ P20 - InSecAirVelocity $2\left(\mathrm{~m} \mathrm{~s}^{\wedge}-1\right)$} & \multicolumn{2}{|c|}{ P21-Mass InSecOxygen 2} & P22-MSWVelocity (m s^-1) & \multicolumn{2}{|c|}{ P5 - coout } & \multicolumn{2}{|c|}{ P6 - Temp Out (K) } & P7 - cosecin \\
\hline 2 & \multicolumn{12}{|c|}{ = Optimization Study } \\
\hline 3 & \multicolumn{2}{|c|}{ Objective } & \multicolumn{2}{|c|}{ No Objective } & \multicolumn{2}{|r|}{ Minimize } & No Objective & \multicolumn{2}{|r|}{ Minimize $\quad \nabla$} & \multicolumn{2}{|c|}{ No Objective $\mathbf{V}$} & \\
\hline 4 & \multirow{2}{*}{\multicolumn{2}{|c|}{ Target Value }} & & & & & \multicolumn{2}{|r|}{ Minimize } & & & No Objective - \\
\hline 5 & & & \multicolumn{2}{|c|}{ Default } & & Higher & Default & \multicolumn{2}{|r|}{ Higher } & \multicolumn{2}{|c|}{ Default } & Default \\
\hline 6 & \multicolumn{12}{|c|}{ - GDO Sample Set 1} \\
\hline 7 & \multicolumn{2}{|c|}{ Candidate A } & - 30,87 & & & 0,20939 & $-0,98001$ & & $-8,0526 \mathrm{E}-06$ & -11 & 104,8 & - 0,090197 \\
\hline 8 & Candi & date B & $-32,79$ & & & 0,21012 & $-0,95367$ & & $-7,9964 \mathrm{E}-06$ & -11 & 104,7 & $-0,090201$ \\
\hline 9 & Candi & date C & $-31,83$ & & & 0,21084 & $-1,0985$ & & $-8,0161 \mathrm{E}-06$ & -11 & 104,8 & $-0,0902$ \\
\hline & $\mathrm{H}$ & & I & J & & $\kappa$ & L & & M & & & $\mathrm{N}$ \\
\hline$P 8-C$ & Secout & $\mathrm{Pg}-\mathrm{Cr}$ & H4Out & P10 - AshTime (s & & P11 - AshTemp (K) & P12 - AshTempMAx & & P13 - O2Out & & $\mathrm{P} 14-\mathrm{COVs}$ & CO2MidRAtio \\
\hline No Ob & jective $\mathbf{\nabla}$ & No Ob & jective $\boldsymbol{\nabla}$ & No Objective & $\boldsymbol{v}$ & Minimize & No Objective & $\checkmark$ & Maximize & $\boldsymbol{\nabla}$ & No Obje & jective \\
\hline & ault & Def & fault & Default & $\nabla$ & Higher & Default & $\nabla$ & Higher & $\nabla$ & Defa & fault \\
\hline-2 & $1433 E-05$ & --2 &, $855 \mathrm{E}-06$ & - 4,9722 & & $-\quad 1174$ & $-1854,5$ & & 故 0,0951 & & $-0,36661$ & \\
\hline-2 & 447E-05 & --2 &, $835 \mathrm{E}-06$ & - 4,9729 & & -1174 & $-1854,3$ & & the 0,0950 & & - 0,36663 & \\
\hline-2 & $632 \mathrm{E}-05$ & --2 & $842 \mathrm{E}-06$ & - 4,9726 & & -1174 & - 1854,4 & & 0,0951 & 101 & $-0,36662$ & \\
\hline
\end{tabular}
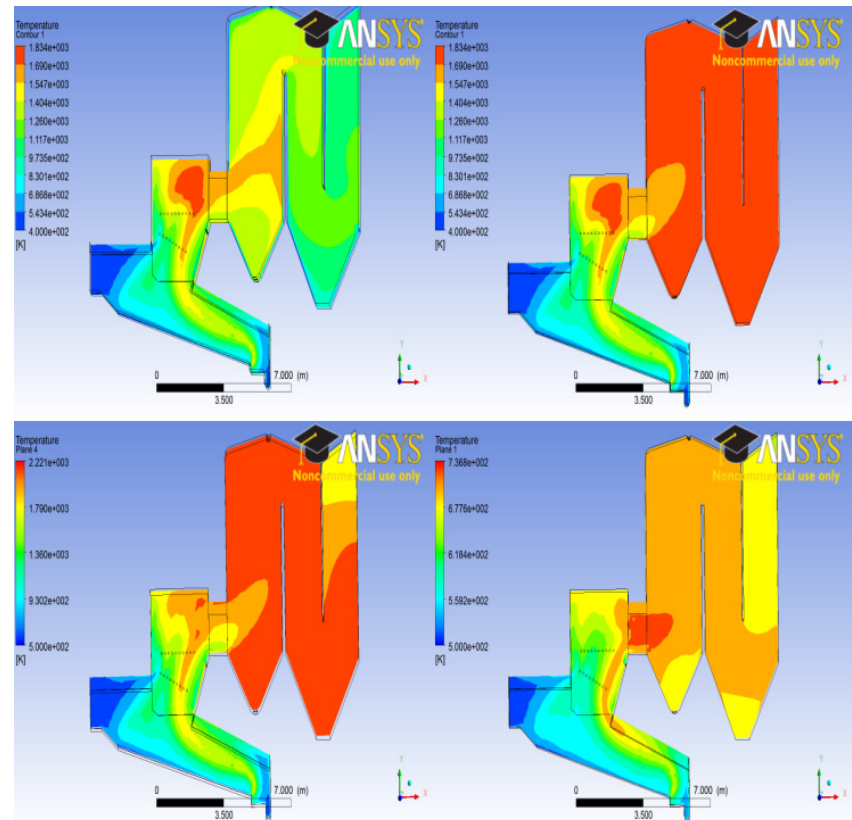

Figure 4: Temperature field by different oxygen MFR at secondary enriched air inlet.

Figure 4 shows the temperature field comparison by different operating condition of oxygen MFR. The temperature in secondary combustion chamber increases when enriched air is used. 


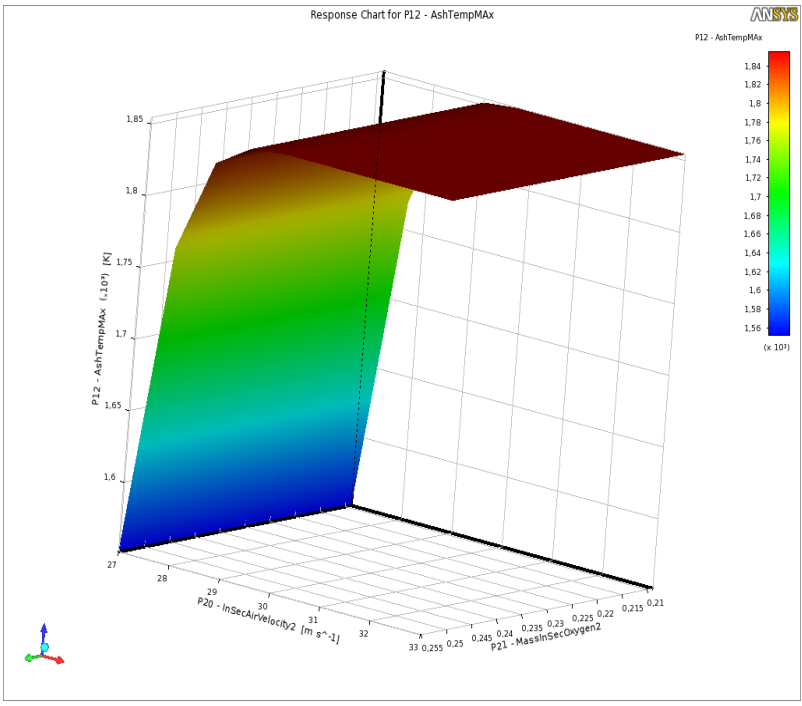

Figure 5: Response chart for maximal ash temperature vs. secondary air velocity and oxygen MFR in secondary air inlet.

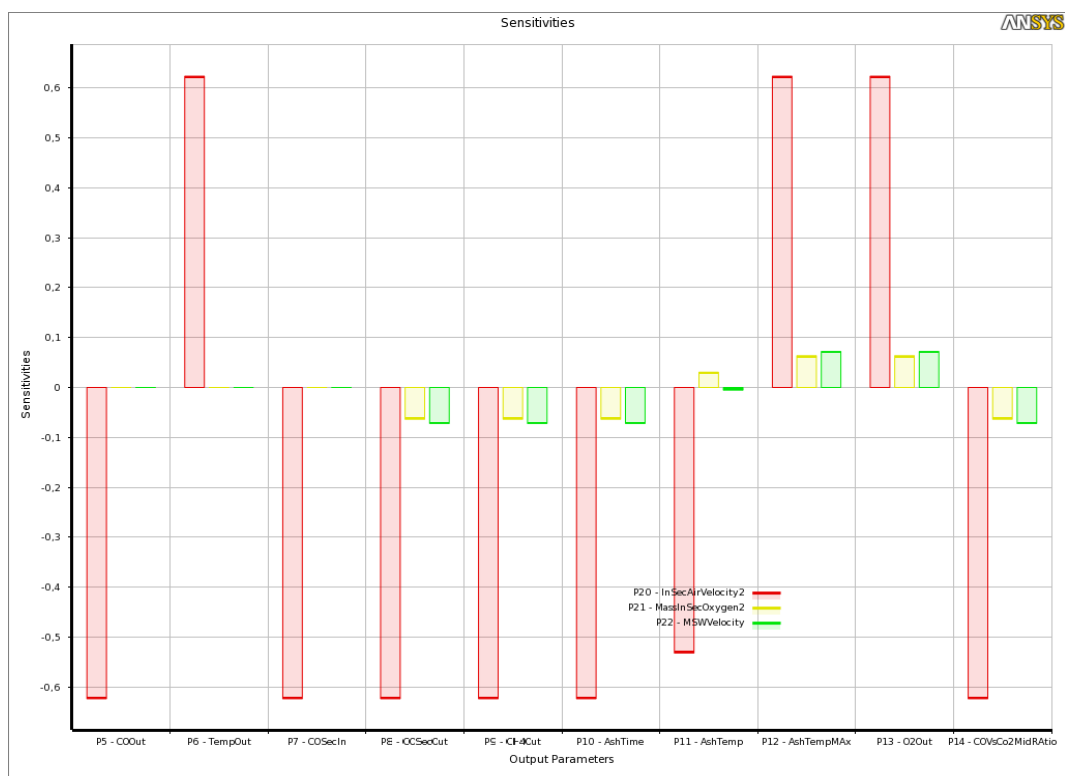

Figure 6: Local parameters sensitivities.

Figure 6 shows the sensitivities among input and output parameters. It is important to know which input parameter has the influence on which output parameter and in which role (negative or positive). As we can see the input 
parameter second air velocity has a huge sensitivity on every output parameters and the other input parameter have the limited sensitivity or no sensitivity on the output parameters.

Figure 7 shows the trade-off study chart for input parameter second air velocity (InSecAirVelocity2) and the output parameter CO at the exit (COOut). The feasible points to match the criteria (Table 1 are shown and dependence of input velocity is very important. For example when the velocity of $27 \mathrm{~m} / \mathrm{s}$ is used the CO MFR at the output is 0.018 and when the velocity of $27.5 \mathrm{~m} / \mathrm{s}$ is used the CO MFR is only 0.0045 .

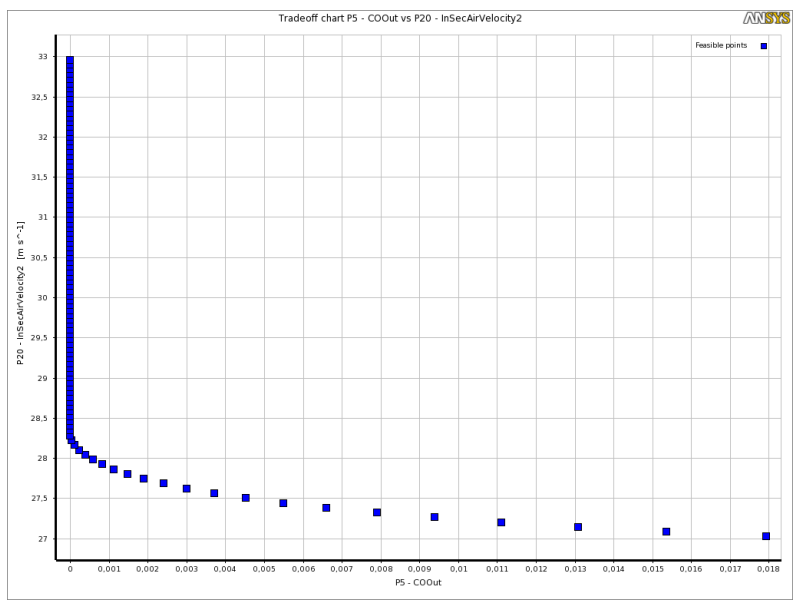

Figure 7: $\quad$ Trade-off chart COOut (OP) vs. second air velocity (IP).

As we can see the CFD is the most convenient tool to predict the optimal conditions which have to be achieved due to the thermal and environmental efficiency and never the less the safety of the WTEP operation. With this tool we can also avoid the problems because we can predict the whole situation with appropriate inlet boundary conditions.

\section{Conclusion}

The applicability of various combustion operating conditions was examined and analyzed, based on the real operating conditions. The CFD approach and the numerical optimisation is used to identify the appropriate conditions to achieve complete combustion and minimize the pollutants emission. The input and output parameters are studied and visualized in the response charts and the local sensitivities among different parameters were presented. Furthermore, the parameters correlation was found out through the parameters matrix. In the end, further simulations have to be done to include the geometry dimensions as input parameters. 


\section{References}

[1] ANSYS, Inc., Southpointe, 275 Technology drive, Canonsburg, PA 15317, United States, Software packet Workbench 2 with CFX 12.0: Help Mode.

[2] ANSYS, Inc., Southpointe, 275 Technology drive, Canonsburg, PA 15317, United States, www.ansys.com/customerportal/index.htm

[3] Chungen Yin, Lasse Rosendahl, Soren H. Kaer, Sonnik Clausen, Soren L. Hvid and Torben Hille, Mathematical modeling and Experimental Study of Biomass Combustion in a Thermal 108 MW Grate-Fired Boiler, Energy \& Fuels, 22, pp. 1380-1390, 2008.

[4] F. Kokalj, N. Samec, An analysis of the combustion conditions in the secondary chamber of a pilot-scale incinerator based on the computational fluid dynamics, Journal of mechanical engineering, 51, pp. 280-303, 2005

[5] Hens-Heinz Frey, Bernhard Peters, Hans Kunsinger, Jürgen Vehlow, Characterization of municipal solid waste combustion in a grate furnace, Waste Management, 23, pp. 689-701, 2003.

[6] M. Costa, M. Dell'Isola, N. Massarotti, Numerical analysis of the thermofluid-dynamic field in the combustion chamber of an incinerator plant, Elsevier, Energy, 34, pp. 2075-2086, 2009.

[7] S.R. Anderson, V. Kadirkamanathan, A. Chipperfield, V. Sharifi, J. Swithenbank, Multi-objective optimization of operating variables in a waste incineration plant, Computer \& chemical Engineering, 29, pp. 1121-1130, 2005.

[8] Won Yang, Hyung-sik Nam, sangmin Choi, improvement of operating conditions in waste incineration using engineering tools, Waste Management, 27, pp. 604-613, 2007.

[9] Yao Bin Yang, Jim Swithenbank, Mathematical modelling of particle mixing effect on the combustion of municipal solid wastes in a packet-bed furnace, Waste Management, 28, pp. 1290-1300, 2008.

[10] Yao Bin Yang, Vida N. Sharifi, Jim Swithenbank, Converting moving-grate incineration from combustion to gasification - Numerical simulation of the burning characteristics, Waste Management, 27, pp. 645-655, 2007.

[11] Y.B. Yang, H. Yamauchi, V. Nasserzadeh, J. Swithenbank, Effects of fuel devolatilisation on the combustion of wood chips and incineration of simulated municipal solid wastes in a packed bed, Fuel, 82, pp. 2205-2221, 2003

[12] Y.B. Yang, Y.B. Goh, R. Zakaria, V. Nasserzadeh, J. Swithenbank, Mathematical modelling of MSW incineration on a travelling bed, Waste management, 22, pp. 369-380, 2002.

[13] Zhaosheng Yu, Xiaoqian Ma,Yanfen Liao, Mathematical modelling of combustion in a grate-fired boiler burning straw and effect of operating conditions under air- and oxygen-enriched atmospheres, Renewable Energy, 35, pp. 895-903, 2010. 